\title{
A Weighted Exponential Detection Function Model for Line Transect Data
}

\author{
Faisal Ababneh \\ Al-Hussian Bin Talal University, Ma'an, Jordan \\ Omar M. Eidous \\ Yarmouk University, Irbid, Jordan
}

Follow this and additional works at: http://digitalcommons.wayne.edu/jmasm

Part of the Applied Statistics Commons, Social and Behavioral Sciences Commons, and the $\underline{\text { Statistical Theory Commons }}$

\section{Recommended Citation}

Ababneh, Faisal and Eidous, Omar M. (2012) "A Weighted Exponential Detection Function Model for Line Transect Data," Journal of Modern Applied Statistical Methods: Vol. 11 : Iss. 1 , Article 11.

DOI: $10.22237 /$ jmasm/1335845400

Available at: http://digitalcommons.wayne.edu/jmasm/vol11/iss1/11

This Regular Article is brought to you for free and open access by the Open Access Journals at DigitalCommons@WayneState. It has been accepted for inclusion in Journal of Modern Applied Statistical Methods by an authorized editor of DigitalCommons@WayneState. 


\title{
A Weighted Exponential Detection Function Model for Line Transect Data
}

\author{
Faisal Ababneh \\ Omar M. Eidous \\ Al-Hussian Bin Talal University, \\ Ma'an, Jordan \\ Yarmouk University, \\ Irbid, Jordan
}

A new parametric model is proposed for modeling the density function of perpendicular distances in line transects sampling. The model can be considered a weighted exponential model in the sense that it combines two exponential models with different weights. The proposed model is appealing because it is monotone decreasing with distance from transect line; in contrast to the classical exponential model, it satisfies the shoulder condition at the origin. Simulation results for a wide range of target densities show reasonable and good performances of the weighted exponential model in most considered cases compared to the classical exponential and the half-normal models.

Key words: Line transect sampling, exponential model, weighted exponential model, half-normal model.

Introduction

Transect methods, particularly line transect methods, are a practical and relatively inexpensive procedure for estimating the population density of certain objects in a given region; these methods have become a popular sampling scheme among ecologists. The estimation procedure can be achieved by walking distance $L$ following a deterministic transect line, counting the number of objects being investigated and recording the perpendicular distance, $X$, from the object sighted to the path of the observer (line transect center). When objects are observed from a line transect with a detection function $g(x)$, the distance $X$ to the observed object from a randomly placed transect will tend to have a probability density function (pdf) $f(x)$ of the same shape as $g(x)$, but scaled so that the area under $f(x)$ equals unity. Buckland, et al.

Omar M. Eidous is a Professor on the Faculty of Science in the Department of Statistics. Email him at: omarm@yu.edu.jo. Faisal Ababneh is Assistant Professor in the Department of Mathematics. Email him at: fmababneh@gmail.com.
(2001) and Burnham, et al. (1980) are the key references for this distance sampling procedure.

The logical assumption related to the detection function $g(x)$ indicates that $g(x)$ is monotonically decreasing and satisfies the shape criterion (i.e., $g^{\prime}(x)=0$ ). Accordingly, $f(x)$ is in turn monotonically decreasing with $f^{\prime}(0)=0$. Burnham and Anderson (1976) gave the fundamental relation for estimating the density of objects in a specific area, which is expressed as

$$
D=\frac{E(n) f(0)}{2 L},
$$

where $E(n)$ is the expected value of the number of detected objects. Given various assumptions (Burnham \& Anderson, 1976) show that the general estimate for $D$ is given by

$$
\hat{D}=\frac{n \hat{f}(0)}{2 L},
$$

where $\hat{f}(0)$ is an appropriate sample estimator of $f(0)$ based on $n$ observed perpendicular distances $x_{1}, x_{2}, \ldots, x_{n}$. Thus, the key aspects in line transects sampling are the modeling of $f(x)$ and the estimation of $f(0)$. 


\section{ABABNEH \& EIDOUS}

Let $f(x)$ be the unknown pdf of perpendicular distances $X_{1}, X_{2}, \ldots, X_{n}$, which are usually assumed to be a random sample (Buckland, et al., 1993). A parametric approach involves assuming that $f(x)$ is a member of a family of proper pdf of a known functional form, but depends on an unknown parameter $\theta$, where $\theta$ may take a vector value and should be estimated by using perpendicular distances. A variety of approaches to estimate $\theta$ will lead to $\hat{f}(0)=f(0, \hat{\theta})$.

A number of parametric models have been proposed for $f(x)$. The classical exponential model and the half normal model, each with one scale parameter, are the most prominent. Gates, et al. (1968) suggested the exponential model with detection function,

$$
g_{1}(x)=e^{-x / \alpha}, \quad x \geq 0
$$

and pdf,

$$
f_{1}(x)=e^{-x / \alpha} / \alpha, \quad x \geq 0 .
$$

The maximum likelihood (ML) method indicates that the ML estimator of $f(0)$ is $\hat{f}_{1, M L}(0)=1 / \bar{X}$, where $\bar{X}$ is the sample mean. The detection function $g_{1}(x)$ (or the pdf $f_{1}(x)$ ) do not satisfy the shoulder condition which minimizes the importance of utilizing this model in line transect sampling. In contrast to the exponential model, the half normal model suggested by Hemingway (1971) satisfies the shoulder condition assumption. The half normal detection function is given by

$$
g_{2}(x)=e^{-x^{2} / 2 \sigma^{2}}, \quad x \geq 0
$$

and the pdf is,

$$
f_{2}(x)=\frac{2}{\sigma \sqrt{2 \pi}} e^{-x^{2} / 2 \sigma^{2}}, x \geq 0
$$

The ML estimator of $f(0)$ is $\hat{f}_{2, M L}(0)=\left(\frac{2}{\pi T}\right)^{1 / 2}$ under the half normal model, where $T=\sum_{i=1}^{n} x_{i}^{2} / n$. Quinn and Gallucci (1980) derived the minimum variance unbiased estimator for $f(0)$ under Model (2), which is given by

$$
\hat{f}_{2, M V}(0)=\frac{1}{\beta(n)}\left(\frac{2}{\pi T}\right)^{1 / 2},
$$

where

$$
\beta(n)=\frac{\Gamma((n-1) / 2)}{\Gamma(n / 2)}\left(\frac{n}{2}\right)^{1 / 2} .
$$

Under Model (2), Zhang (2009) proposed the shrinkage estimator

$$
\hat{f}_{2, S H}(0)=\frac{n-2}{n} \beta(n)\left(\frac{2}{\pi T}\right)^{1 / 2} .
$$

The estimator $\hat{f}_{2, S H}(0)$ is biased for $f(0)$, but it achieves the smallest mean square error.

Comparing the estimators $\hat{f}_{2, M L}(0), \hat{f}_{2, M V}(0)$ and $\hat{f}_{2, S H}(0)$ it is observed that

$$
\begin{aligned}
\hat{f}_{2, S H}(0) & =\frac{n-2}{n} \beta^{2}(n) \hat{f}_{2, M V}(0) \\
& =\frac{n-2}{n} \beta(n) \hat{f}_{2, M L}(0)
\end{aligned}
$$

Because $\beta(n) \rightarrow 1$ (Magnus, et al., 1966) and $\frac{n-2}{n} \rightarrow 1$ as $n \rightarrow \infty$, the three estimators are asymptotically equivalent. A simulation study was performed using a finite sample for different target models; results indicate that the three estimators perform very similarly to each other, even for the target detection functions that are deviated from the half normal model.(For other parametric models with two parameters see: Burnham \& Anderson (1976); Pollock, (1978); 


\section{WEIGHTED EXPONENTIAL DETECTION MODEL FOR LINE TRANSECT DATA}

Burnham, et al. (1980); Buckland (1985); Eidous (2004).

A weighted exponential model with one parameter is proposed to fit line transects data. Two estimators under this model are derived to estimate $f(0)$ and hence the density of objects, $D$. The small-sample properties of the new estimators were studied and compared to both the classical exponential and the half normal estimators via simulation techniques.

The Model

Let $X_{1}, X_{2}, \ldots, X_{n}$ be $n$ perpendicular distances (assumed to be independent and identically distributed) following the detection function $g(x ; \theta, \gamma)$, where $\theta$ and $\gamma$ are two unknown parameters. The detection function is proposed to be,

$$
\begin{aligned}
& g(x ; \theta, \gamma)=2 e^{-\theta x}-e^{-\lambda x}, \\
& x \geq 0, \theta, \gamma>0
\end{aligned}
$$

According to this detection function, the probability of detecting an object given its perpendicular distance on the transect line is one (i.e., $g(0 ; \theta, \gamma)=1)$, which indicates that the probability of detecting on the line transect center is certain. However, the first derivative of $g(x ; \theta, \gamma)$ at $x=0$ is $-2 \theta+\gamma \quad$ (i.e. $\left.g^{\prime}(0 ; \theta, \gamma)=-2 \theta+\gamma\right)$, which indicates that $g(x ; \theta, \gamma)$ do not satisfy the shoulder condition unless $\gamma=2 \theta$. Therefore, the detection function for the perpendicular distances that satisfies the shoulder condition is proposed to be,

$$
g_{3}(x)=e^{-\theta x}\left(2-e^{-\theta x}\right),
$$

and the corresponding pdf is,

$$
f_{3}(x)=\frac{2}{3 \theta} e^{-\theta x}\left(2-e^{-\theta x}\right), \quad x \geq 0, \theta>0 .
$$

Because $g_{3}(0)=1$, the parameter $f_{3}(0)$ is given by

$$
f_{3}(0)=\frac{2}{3 \theta}
$$

model (3) can be expressed in terms of $f_{3}(0)$ as

$$
\begin{aligned}
& f_{3}(x)=f_{3}(0) e^{-3 f_{3}(0) x / 2}\left(2-e^{-3 f_{3}(0) x / 2}\right), \\
& x \geq 0
\end{aligned}
$$

It can be shown that the detection function $g_{3}(x)$ is monotonically decreasing in $x$. The first derivative of $g_{3}(x)$ is $g_{3}^{\prime}(x)=2 \theta\left(e^{-2 \theta x}-e^{-\theta x}\right)$, which equals zero at $x=0 ;$ thus, $g_{3}(x)$ is monotonically decreasing for $x \in(0, \infty)$ if $2 \theta\left(e^{-2 \theta x}-e^{-\theta x}\right)$ $<0 \Rightarrow e^{-2 \theta x}-e^{-\theta x}<0 \Rightarrow e^{-2 \theta x}<e^{-\theta x} \Rightarrow$ $-2 \theta x<-\theta x \Rightarrow 2 \theta x>\theta x$, which is true for all $x>0$ and $\theta>0$. Accordingly $f_{3}(x)$ is monotonically decreasing for $x \in(0, \infty)$.

Moments and Maximum Likelihood Estimators The parameter $f_{3}(0)$ in model (5) must be estimated. The expected value of $X$ based on this model is $7 /\left(9 f_{3}(0)\right)$, which gives $\hat{f}_{3, M O}(0)=7 /(9 \bar{X})$ as the moment estimator for $f_{3}(0)$.

Although the moment estimator for $f_{3}(0)$ is given in a closed form, the maximum likelihood estimator must be calculated using a numerical method. The likelihood function $L(f)$ based on model (5) is

$L(f)=\prod_{i=1}^{n} f_{3}\left(x_{i}\right)$

$=f_{3}(0)^{n} \exp \left(-3 f_{3}(0) \sum_{\mathrm{i}=1}^{\mathrm{n}} \frac{\mathrm{x}_{\mathrm{i}}}{2}\right) \prod_{\mathrm{i}=1}^{\mathrm{n}}\left(2-\exp \left(\frac{-3 f_{3}(0) \mathrm{x}_{\mathrm{i}}}{2}\right)\right)$.

To find the maximum likelihood estimator of $f_{3}(0)$, the following equation must be solved

$$
\frac{\partial \log L(f)}{\partial f}=0
$$

where 
$\frac{\partial \log L(f)}{\partial f}=$

$\frac{n}{f_{3}(0)}-\frac{3}{2} \sum_{i=1}^{n} x_{i}+\frac{3}{2} \sum_{i=1}^{n}\left(\frac{x_{i}}{2 \exp \left(3 f_{3}(0) x_{i} / 2\right)-1}\right)$.

The maximum likelihood estimator can be found by using numerical methods such as the Newton-Raphson, and a Mathematica Program for carrying out the estimation procedure can be written. Let $\hat{f}_{3, M L}(0)$ be the maximum likelihood estimator of $f_{3}(0)$, then as $n \rightarrow \infty$, $\hat{f}_{3, M L}(0)$ is asymptotically $N\left(f_{3}(0), \sigma_{f}^{2}\right)$, where $\sigma_{f}^{2}=\left\{-E\left(\frac{d^{2} \ln L(f)}{d f^{2}}\right)\right\}^{-1}$. Using the transformation $\quad u=2-\exp \left(-3 f_{3}(0) x / 2\right)$ results in,

$$
\begin{aligned}
& -E\left(\frac{d^{2} \ln L(f)}{d f^{2}}\right) \\
& =\frac{n}{f_{3}(0)^{2}}+\frac{9}{2} \sum_{i=1}^{n} E\left(\frac{X_{i}^{2} \exp \left(-3 f_{3}(0) X_{i} / 2\right)}{\left(2-\exp \left(-3 f_{3}(0) X_{i} / 2\right)\right)^{2}}\right) \\
& =\frac{n}{f_{3}(0)^{2}}+\frac{4 n}{3 f_{3}(0)^{2}} \int_{1}^{2} \frac{(2-u) \ln ^{2}(2-u)}{u} d u
\end{aligned}
$$

The Mathematica Program was used to compute the last integral, which gives,

$$
=\frac{0.80153 n}{f_{3}(0)^{2}} \text {. }
$$

Therefore,

$$
\sigma_{f}^{2}=\frac{f_{3}(0)^{2}}{0.80153 n}
$$

Replacing $f_{3}(0)$ by $\hat{f}_{3, M L}(0)$ leads to the estimate $\hat{\sigma}_{f}^{2}$ of $\sigma_{f}^{2}$ and the approximate largesample $(1-\alpha) 100 \%$ confidence interval (C.I.) for $f_{3}(0)$ is given by

$$
\hat{f}_{3, M L}(0) \pm Z_{\alpha / 2} \sqrt{\hat{\sigma}_{f}^{2}}
$$

For example, if $\alpha=0.05$, then $Z_{0.025}=1.96$ and thus, a $95 \%$ C.I. for $f_{3}(0)$ is $\hat{f}_{3, M L}(0) \pm$ $2.18925 \hat{f}_{3, M L}(0) / \sqrt{n}$.

\section{Methodology}

To assess the performances of the proposed estimators $\hat{f}_{3, M O}(0)$ and $\hat{f}_{3, M L}(0)$ of $f(0)$ under the weighted exponential family, a simulation study was performed. For comparison, the classical exponential estimator $\hat{f}_{1, M L}(0)$ and the half normal estimator $\hat{f}_{2, M L}(0)$ were also considered. Four target models were chosen for inclusion in the simulation based on the criterion that they are representative of many different shapes that might occur in the field. These four models are:

(1) Exponential Power (EP) Model (Pollock, 1978)

$$
\begin{aligned}
& f(x)=\frac{1}{\Gamma(1+1 / \beta)} e^{-x^{\beta}} \\
& x \geq 0, \quad \beta \geq 1
\end{aligned}
$$

(2) Hazard-Rate (HR) Model (Hayes and Buckland, 1983)

$$
\begin{aligned}
& f(x)=\frac{1}{\Gamma(1-1 / \beta)}\left(1-e^{-x^{-\beta}}\right) \\
& x \geq 0, \quad \beta>1
\end{aligned}
$$

(3) Beta (BE) Model (Eberhardt, 1968)

$$
\begin{aligned}
& f(x)=(1+\beta)(1-x)^{\beta} \\
& 0 \leq x<1, \beta \geq 0
\end{aligned}
$$

(4) General Polynomial (GP) Model (Zhang, 2009)

$$
\begin{aligned}
& f(x)=\frac{10 \Gamma(\beta)}{3 \sqrt{\pi} \Gamma(\beta-1 / 2)}\left(1+(x / 0.6)^{2}\right)^{-\beta} \\
& x \geq 0, \beta>1 / 2 .
\end{aligned}
$$




\section{WEIGHTED EXPONENTIAL DETECTION MODEL FOR LINE TRANSECT DATA}

Four models were selected from the following different models: EP, HR, BE and GP. For the EP model, parameter values $\beta=1.0,1.5,2.0,2.5$ and corresponding truncation points $w=5.0,3.0,2.5,2.0$. For the HR model with parameter values $\beta=1.5,2.0,2.5,3.0$ and corresponding truncation points $w=20,12,8,6$. The $\mathrm{BE}$ model parameter values $\beta=1.5,2.0,2.5,3.0$ and truncated point $w=1.0$ for all cases. For the GP model parameter values $\beta=0.6,0.9,1.9,3.5$ and the truncated point $w=3.0$ for all cases.

The 16 target models considered cover a wide range of perpendicular distance probability density functions that vary near zero from spike to flat. It should be noted that the EP model with $\beta=1$ and the $\mathrm{BE}$ model with different values of $\beta$ do not satisfy the shoulder condition assumption. These choices were made in order to assess the robustness of the considered estimators with respect to the violation of the shoulder condition assumption. Note also that the other considered models satisfy the shoulder condition assumption.

For each model 1,000 samples of perpendicular distances were randomly drawn for sample sizes $n=50,100,200$. Table (1) reports the simulated value of the relative bias $(R B)$ for each model and for each sample size,

$$
R B=\frac{E(\hat{f}(0))-f(0)}{f(0)},
$$

and the relative mean error $(R M E)$

$$
R M E=\frac{\sqrt{M S E(\hat{f}(0))}}{f(0)},
$$

for each considered estimator.

\section{Conclusion}

Based on the simulation, several conclusions can be drawn by inspecting the results with respect to $R B$ and $R M E$.
(1) The performance of the classical exponential estimator, $\hat{f}_{1, M L}(0)$ is effective when the target (underlying) model is exponential (EP with $\beta=1$ ). In this case, the $R B$ and $R M E$ associated with $\hat{f}_{1, M L}(0)$ were very small compared other considered estimators. However, the $R B$ and $R M E$ of $\hat{f}_{1, M L}(0)$ become very large when the underlying model deviates from the exponential. The $R M E$ values of $\hat{f}_{1, M L}(0)$ range between 0.080 (EP with $\beta=1$ and $n=200$ ) and 0.732 (EP with $\beta=2.5$ and $n=50$ ).

(2) The estimator $\hat{f}_{2, M L}(0)$ seems to be better than $\hat{f}_{1, M L}(0)$ for most considered cases. Regarding $R M E, \quad \hat{f}_{2, M L}(0)$ beats $\hat{f}_{1, M L}(0)$ for all considered cases except for (EP with $\beta=1$ and HR with $\beta=1.5$ and $\beta=2$ ). Despite that HR satisfies the shoulder condition, it decreases very sharply away $x=0$ when $\beta=1.5$ and $\beta=2$. This may explain the performances of $\hat{f}_{1, M L}(0)$ in these two cases. The performance of $\hat{f}_{2, M L}(0)$ is very good when the target model is half normal (EP with $\beta=2$ ) and when the shoulder condition of the target model is very large (EP with $\beta=2.5$ and HR with $\beta=3$ ). Except for the cases: EP with $\beta=1$, HR with $\beta=1.5$ and $\beta=2$, the performance of $\hat{f}_{2, M L}(0)$ is acceptable compared the other estimators. The RME values of $\hat{f}_{2, M L}(0)$ range from 0.052 (EP with $\beta=2$ and $n=200$ ) to 0.576 (EP with $\beta=1.5$ and $n=200$ ). 


\section{ABABNEH \& EIDOUS}

(3) The $R B$ and $R M E$ associated with the two proposed estimators $\hat{f}_{3, M O}(0)$ and $\hat{f}_{3, M L}(0)$ are very similar to each other in all considered cases. Therefore, $\hat{f}_{3, M O}(0)$ is recommended as opposed to $\hat{f}_{3, M L}(0)$ because the formal model takes a closed form, while $\hat{f}_{3, M L}(0)$ needs a numerical method to compute. Comparing the RME $\mathrm{s}$ of $\hat{f}_{3, M O}(0)$ and $\hat{f}_{2, M L}(0)$ it appears that $\hat{f}_{3, M O}(0)$ performs better than $\hat{f}_{2, M L}(0)$ in most cases. More specifically, if the shoulder condition of the target model seems to be large, then $\hat{f}_{2, M L}(0)$ beats $\hat{f}_{3, M O}(0)$ (e.g. EP with $\beta=2,2.5$ and HR with $\beta=3)$. Otherwise, $\hat{f}_{3, M O}(0)$ performs better than $\hat{f}_{2, M L}(0)$. The $R M E$ values of $\hat{f}_{3, M O}(0)$ range from 0.058 (GP with $\beta=0.6$ and $n=200$ ) to 0.357 (EP with $\beta=2.5$ and $n=50)$. Comparing the range of $R M E$ for different estimators indicates that $\hat{f}_{3, M O}(0)$ is more stable than the other estimators.

Therefore, based on results in this study, it may be concluded that the weighted exponential model fits the line transect data reasonably and it can be recommended as a promising parametric model to estimate the parameter $f(0)$ and the population density $D$.

\section{References}

Bain, L. J., \& Engelhardt, M. (1992).Introduction to probability and mathematical statistics, $2^{\text {nd }} E d$. Boston, MA: Duxbury Press.
Barabesi, L. (2001). Local parametric density estimation methods in line transect sampling. Metron, LIX, 21-37.

Buckland, S. T. (1985). Perpendicular distance models for line transect sampling. Biometrics, 41, 177-195.

Buckland, S. T. (1992). Fitting density functions using polynomials. Applied Statistics, 41, 63-76.

Buckland, S. T., Anderson, D. R., Burnham, K. P., Laake, J. L., Borchers, D. L., \& Thomas, L. (2001). Introduction to distance sampling. Oxford, England: Oxford University Press.

Burnham, K. P., \& Anderson, D. R. (1976). Mathematical models for nonparametric inferences from line transect data. Biometrics, 32, 325-336.

Burnham, K. P., Anderson, D. R., \& Laake, J. L. (1980). Estimation of density from line transect sampling of biological populations. Wildlife Monograph, No. 72, 1-202.

Chen, S. X. (1996). A kernel estimate for the density of a biological population by using line transect sampling. Applied Statistics, 45, 135-150.

Eidous, O. M. (2004). A parametric family for density estimation in line transect sampling. Abhath Al-Yarmouk, 13, 315-326.

Gates, C. E., Marshall, W. H., \& Olson, D. P. (1968).Line transect method of estimating grouse population densities. Biometrics, 24, 135145.

Hayes, R. J., \& Buckland, S. T. (1983). Radial distance models for line-transect method. Biometrics, 39, 29-42.

Magnus, W., Oberhettinger, F., \&Soni, R. P. (1966).Formulas and theorems for special functions of mathematical physics. New York, NY: Springer-Verlag.

Pollock, K. H. (1978). A family of density estimators for line transect sampling. Biometrics, 34, 475-478.

Zhang, S. (2009). On parametric estimation of population abundance for line transect sampling. Environmental and Ecological Statistics, 18, 79-92. 
WEIGHTED EXPONENTIAL DETECTION MODEL FOR LINE TRANSECT DATA

Table 1: RB and RME for Different Estimators when Data are Simulated from the Four Target Models

\begin{tabular}{|c|c|c|c|c|c|c|c|c|c|c|}
\hline \multirow[b]{3}{*}{$n$} & \multirow[b]{3}{*}{$\beta$} & \multirow[b]{3}{*}{$w$} & \multicolumn{8}{|c|}{ Estimator } \\
\hline & & & \multicolumn{2}{|c|}{$\hat{f}_{1, M L}(0)$} & \multicolumn{2}{|c|}{$\hat{f}_{2, M L}(0)$} & \multicolumn{2}{|c|}{$\hat{f}_{3, M O}(0)$} & \multicolumn{2}{|c|}{$\hat{f}_{3, M L}(0)$} \\
\hline & & & $\mathrm{RB}$ & RME & $\mathrm{RB}$ & RME & $\mathrm{RB}$ & RME & $\mathrm{RB}$ & RME \\
\hline \multicolumn{11}{|c|}{ Exponential Power (EP) Model } \\
\hline 50 & \multirow{3}{*}{1} & \multirow{3}{*}{5} & 0.055 & 0.154 & -0.384 & 0.393 & -0.179 & 0.211 & -0.187 & 0.219 \\
\hline 100 & & & 0.040 & 0.105 & -0.393 & 0.397 & -0.191 & 0.206 & -0.187 & 0.204 \\
\hline 200 & & & 0.041 & 0.080 & -0.395 & 0.397 & -0.190 & 0.198 & -0.186 & 0.199 \\
\hline 50 & \multirow{3}{*}{1.5} & \multirow{3}{*}{3} & 0.400 & 0.435 & -0.133 & 0.166 & 0.089 & 0.159 & 0.089 & 0.155 \\
\hline 100 & & & 0.391 & 0.408 & -0.141 & 0.156 & 0.082 & 0.122 & 0.081 & 0.118 \\
\hline 200 & & & 0.390 & 0.399 & -0.142 & 0.149 & 0.081 & 0.103 & 0.078 & 0.102 \\
\hline 50 & \multirow{3}{*}{2} & \multirow{3}{*}{2.5} & 0.593 & 0.617 & 0.017 & 0.104 & 0.239 & 0.274 & 0.248 & 0.281 \\
\hline 100 & & & 0.590 & 0.602 & 0.015 & 0.073 & 0.236 & 0.254 & 0.243 & 0.261 \\
\hline 200 & & & 0.580 & 0.586 & 0.008 & 0.052 & 0.229 & 0.238 & 0.233 & 0.242 \\
\hline 50 & \multirow{3}{*}{2.5} & \multirow{3}{*}{2} & 0.711 & 0.732 & 0.118 & 0.155 & 0.331 & 0.357 & 0.345 & 0.373 \\
\hline 100 & & & 0.697 & 0.708 & 0.109 & 0.132 & 0.320 & 0.334 & 0.330 & 0.343 \\
\hline 200 & & & 0.695 & 0.700 & 0.105 & 0.117 & 0.318 & 0.325 & 0.326 & 0.331 \\
\hline \multicolumn{11}{|c|}{ Hazard Rate (HR) Model } \\
\hline 50 & \multirow{3}{*}{1.5} & \multirow{3}{*}{20} & -0.120 & 0.199 & -0.565 & 0.571 & -0.316 & 0.339 & -0.311 & 0.337 \\
\hline 100 & & & -0.126 & 0.169 & -0.570 & 0.573 & -0.320 & 0.332 & -0.310 & 0.335 \\
\hline 200 & & & -0.131 & 0.152 & -0.575 & 0.576 & -0.324 & 0.329 & -0.323 & 0.331 \\
\hline 50 & \multirow{3}{*}{2} & \multirow{3}{*}{12} & 0.149 & 0.256 & -0.409 & 0.427 & -0.107 & 0.194 & -0.128 & 0.215 \\
\hline 100 & & & 0.126 & 0.187 & -0.427 & 0.434 & -0.124 & 0.164 & -0.126 & 0.175 \\
\hline 200 & & & 0.112 & 0.152 & -0.437 & 0.441 & -0.135 & 0.157 & -0.127 & 0.149 \\
\hline 50 & \multirow{3}{*}{2.5} & \multirow{3}{*}{8} & 0.387 & 0.443 & -0.226 & 0.270 & 0.079 & 0.186 & 0.067 & 0.161 \\
\hline 100 & & & 0.394 & 0.421 & -0.231 & 0.252 & 0.084 & 0.143 & 0.083 & 0.139 \\
\hline 200 & & & 0.374 & 0.386 & -0.250 & 0.258 & 0.068 & 0.102 & 0.069 & 0.109 \\
\hline 50 & \multirow{3}{*}{3} & \multirow{3}{*}{6} & 0.559 & 0.599 & -0.074 & 0.169 & 0.213 & 0.270 & 0.236 & 0.294 \\
\hline 100 & & & 0.559 & 0.579 & -0.084 & 0.135 & 0.213 & 0.242 & 0.226 & 0.255 \\
\hline 200 & & & 0.543 & 0.552 & -0.099 & 0.122 & 0.200 & 0.215 & 0.213 & 0.227 \\
\hline
\end{tabular}


ABABNEH \& EIDOUS

Table 1 (continued): RB and RME for Different Estimators when Data are Simulated from the Four Target Models

\begin{tabular}{|c|c|c|c|c|c|c|c|c|c|c|}
\hline \multirow[b]{3}{*}{$n$} & \multirow[b]{3}{*}{$\beta$} & \multirow[b]{3}{*}{$w$} & \multicolumn{8}{|c|}{ Estimator } \\
\hline & & & \multicolumn{2}{|c|}{$\hat{f}_{1, M L}(0)$} & \multicolumn{2}{|c|}{$\hat{f}_{2, M L}(0)$} & \multicolumn{2}{|c|}{$\hat{f}_{3, M O}(0)$} & \multicolumn{2}{|c|}{$\hat{f}_{3, M L}(0)$} \\
\hline & & & $\mathrm{RB}$ & RME & $\mathrm{RB}$ & RME & $\mathrm{RB}$ & RME & $\mathrm{RB}$ & RME \\
\hline \multicolumn{11}{|c|}{ Beta (BE) Model } \\
\hline 50 & \multirow{3}{*}{1.5} & \multirow{3}{*}{1} & 0.410 & 0.436 & -0.096 & 0.126 & 0.096 & 0.151 & 0.092 & 0.152 \\
\hline 100 & & & 0.406 & 0.419 & -0.099 & 0.115 & 0.093 & 0.124 & 0.103 & 0.134 \\
\hline 200 & & & 0.403 & 0.410 & -0.101 & 0.109 & 0.092 & 0.107 & 0.096 & 0.109 \\
\hline 50 & \multirow{3}{*}{2} & \multirow{3}{*}{1} & 0.350 & 0.382 & -0.148 & 0.171 & 0.050 & 0.130 & 0.067 & 0.134 \\
\hline 100 & & & 0.336 & 0.352 & -0.157 & 0.168 & 0.039 & 0.091 & 0.046 & 0.093 \\
\hline 200 & & & 0.332 & 0.340 & -0.158 & 0.163 & 0.036 & 0.066 & 0.041 & 0.072 \\
\hline 50 & \multirow{3}{*}{2.5} & \multirow{3}{*}{1} & 0.297 & 0.332 & -0.189 & 0.207 & 0.009 & 0.117 & 0.021 & 0.121 \\
\hline 100 & & & 0.298 & 0.315 & -0.189 & 0.198 & 0.010 & 0.080 & 0.010 & 0.075 \\
\hline 200 & & & 0.289 & 0.298 & -0.196 & 0.200 & 0.003 & 0.057 & -0.000 & 0.054 \\
\hline 50 & \multirow{3}{*}{3} & \multirow{3}{*}{1} & 0.263 & 0.301 & -0.216 & 0.231 & -0.018 & 0.115 & -0.011 & 0.116 \\
\hline 100 & & & 0.257 & 0.276 & -0.221 & 0.229 & -0.023 & 0.082 & -0.023 & 0.080 \\
\hline 200 & & & 0.256 & 0.267 & -0.223 & 0.227 & -0.023 & 0.061 & -0.025 & 0.060 \\
\hline \multicolumn{11}{|c|}{ General Polynomial (GP) Model } \\
\hline 50 & \multirow{3}{*}{0.6} & \multirow{3}{*}{3} & 0.254 & 0.290 & -0.220 & 0.243 & 0.030 & 0.117 & 0.033 & 0.123 \\
\hline 100 & & & 0.245 & 0.262 & -0.233 & 0.238 & 0.022 & 0.083 & 0.021 & 0.073 \\
\hline 200 & & & 0.246 & 0.259 & -0.217 & 0.229 & 0.018 & 0.058 & 0.014 & 0.061 \\
\hline 50 & \multirow{3}{*}{0.9} & \multirow{3}{*}{3} & 0.189 & 0.243 & -0.296 & 0.308 & -0.075 & 0.141 & -0.077 & 0.154 \\
\hline 100 & & & 0.181 & 0.211 & -0.302 & 0.308 & -0.081 & 0.117 & -0.095 & 0.128 \\
\hline 200 & & & 0.170 & 0.187 & -0.309 & 0.311 & -0.090 & 0.108 & -0.092 & 0.110 \\
\hline 50 & \multirow{3}{*}{1.9} & \multirow{3}{*}{3} & 0.280 & 0.332 & -0.262 & 0.289 & -0.004 & 0.138 & 0.001 & 0.141 \\
\hline 100 & & & 0.275 & 0.303 & -0.276 & 0.289 & -0.009 & 0.099 & -0.011 & 0.101 \\
\hline 200 & & & 0.267 & 0.283 & -0.284 & 0.291 & -0.015 & 0.074 & -0.019 & 0.075 \\
\hline 50 & \multirow{3}{*}{3.5} & \multirow{3}{*}{3} & 0.452 & 0.489 & -0.118 & 0.174 & 0.129 & 0.194 & 0.121 & 0.191 \\
\hline 100 & & & 0.435 & 0.454 & -0.135 & 0.163 & 0.116 & 0.154 & 0.119 & 0.155 \\
\hline 200 & & & 0.426 & 0.436 & -0.140 & 0.154 & 0.109 & 0.130 & 0.120 & 0.142 \\
\hline
\end{tabular}

